\title{
Fungal glycosyl hydrolases for sustainable plant biomass valorization: Talaromyces amestolkiae as a model fungus
}

\author{
Alicia Prieto ${ }^{1}$ - Laura de Eugenio ${ }^{1} \cdot$ Juan A. Méndez-Líter ${ }^{1} \cdot$ Manuel Nieto-Domínguez $^{1} \cdot$ Carlos Murgiondo $^{1}$. \\ Jorge Barriuso ${ }^{1} \cdot$ Lara Bejarano-Muñoz $^{1}$ - María Jesús Martínez ${ }^{1}$ (I)
}

Received: 2 June 2021 / Revised: 3 August 2021 / Accepted: 4 August 2021

(C) The Author(s), under exclusive licence to Springer Nature Switzerland AG 2021

\begin{abstract}
As the main decomposers and recyclers in nature, fungi secrete complex mixtures of extracellular enzymes for degradation of plant biomass, which is essential for mobilization of the organic carbon fixed by the photosynthesis in vegetal cells. Biotechnology can emulate the closed natural biological cycles, using lignocellulosic biomass as a renewable resource and lignocellulolytic fungal enzymes as catalysts to sustainably produce consumer goods. Cellulose and hemicellulose are the major polysaccharides on Earth, and the main enzymes involved in their hydrolytic depolymerization are cellulases (endoglucanases, cellobiohydrolases, and $\beta$-glucosidases) and hemicellulases (mainly endoxylanases and $\beta$-xylosidases). This work will focus on the enzymes secreted by the filamentous ascomycete Talaromyces amestolkiae and on some of their biotechnological applications. Their excellent hydrolytic activity was demonstrated by the partial degradation of xylans to prebiotic oligosaccharides by the endoxylanase $\mathrm{XynN}$, or by the saccharification of lignocellulosic wastes to monosaccharides (fermentable to ethanol) either by the whole secretomes or by isolated enzymes used as supplements of commercial cocktails. However, apart from their expected hydrolytic activity, some of the $\beta$-glycosidases produced by this strain catalyze the transfer of a sugar molecule to specific aglycons by transglycosylation. As the synthesis of customized glycoconjugates is a major goal for biocatalysis, mutant variants of the $\beta$-xyloxidase BxTW1 and the $\beta$-glucosidases BGL-1 and BGL-2 were obtained by directed mutagenesis, substantially improving the regioselective production yields of bioactive glycosides since they showed reduced or null hydrolytic activity.
\end{abstract}

Keywords Ascomycete $\cdot$ Cellulases $\cdot$ Xylanases $\cdot$ Bioethanol $\cdot$ Bioactive compounds

Human activity is unavoidably linked to the consumption of goods and services and, therefore, to the productive sector. Over several decades and driven by the seemingly endless number of materials and products derived from the petrochemical industry, the economy has been based on a linear production scheme, represented by the take-make-waste extractive industrial model (Geissdoerfer et al. 2017). However, the time has put on the table compelling reasons to change our productive mind-sets and consumption habits. Fossil resources used as raw materials are finite, forcing the development of new processes based on the transformation

Alicia Prieto

aliprieto@cib.csic.es

$\triangle$ María Jesús Martínez

mjmartinez@cib.csic.es

1 Centro de Investigaciones Biológicas Margarita Salas (CIBCSIC), C/ Ramiro de Maeztu 9, 28022 Madrid, Spain of renewable resources into current materials and products, or new ones that meet the same needs as existing ones, but without a doubt, the most negative effect derived from the existing production and consumption systems, established over the last century, has been its environmental impact. The multitude of non-biodegradable waste accumulated in soils and waters, air pollution, land impoverishment and the generation of harmful gases resulting from agricultural and livestock overexploitation are causing tremendous damage to all ecosystems and decisively affecting the climate. All this has stimulated collective awareness and driven a paradigm shift towards what has been called the circular economy. According to the European Environmental Agency, this involves the gradual use of renewable or recyclable resources, reducing the consumption of raw materials and energy, protecting the environment through cutting emissions and minimizing material losses and, thus, waste generation (European Commission, 2015). This scheme emulates what occurs in 
biogeological cycles in the biosphere (European Environmental Agency, 2018), in which recycling is essential to maintain the equilibrium that supports life.

In the scheme of circular economy, biomass appears as the most promising material for biotransformations (Ubando et al. 2020). Plant biomass is considered the main reservoir of organic carbon in terrestrial ecosystems (Bar-On et al. 2018) and, as such, the search of efficient processes for its exploitation as a renewable source of energy and raw materials has been among the priority research areas for several decades. In fact, the need to develop new energy sources not based on fossil fuels within the transport sector triggered extensive research in biomass-derived liquid biofuels (Aditiya et al. 2016). The so-called first-generation bioethanol uses easily degradable carbohydrates (sucrose, starch) to obtain ethanol from microbial fermentation of glucose. Although this is the currently implemented technology, this process has proven not to be appropriate to maintain a sustainable and non-polluting production of essential goods, which has promoted the exploitation of lignocellulosic sugars (especially agricultural wastes) as feedstock to produce the denominated second-generation ethanol (Robak and Balcerek 2018). Given the complex structure of lignocellulose, obtaining fermentable sugars from such material has been a major challenge.

In this context, biotechnological approaches have helped to develop cleaner and less energy-demanding industrial processes than those based only on chemical methods. Enzymes and microorganisms are used within biorefineries, defined as facilities that integrate biomass conversion processes and equipment to produce fuels, power, and value-added chemicals from biomass (Demirbas, 2010).

\section{Composition of plant biomass and role of fungi in its degradation}

As defined by the IUPAC, biomass is defined as "material produced by the growth of microorganisms, plants, or animals." The organic carbon content of the total biomass in the biosphere has been estimated at about 550 gigatons of carbon and, as the dominant kingdom, the contribution of plants to this figure exceeds $80 \%$, making plant biomass the main natural resource (Bar-On et al. 2018). In plants, the organic carbon is mostly contained in the three major components of plant cell walls: cellulose, hemicellulose, and lignin (Fig. 1).

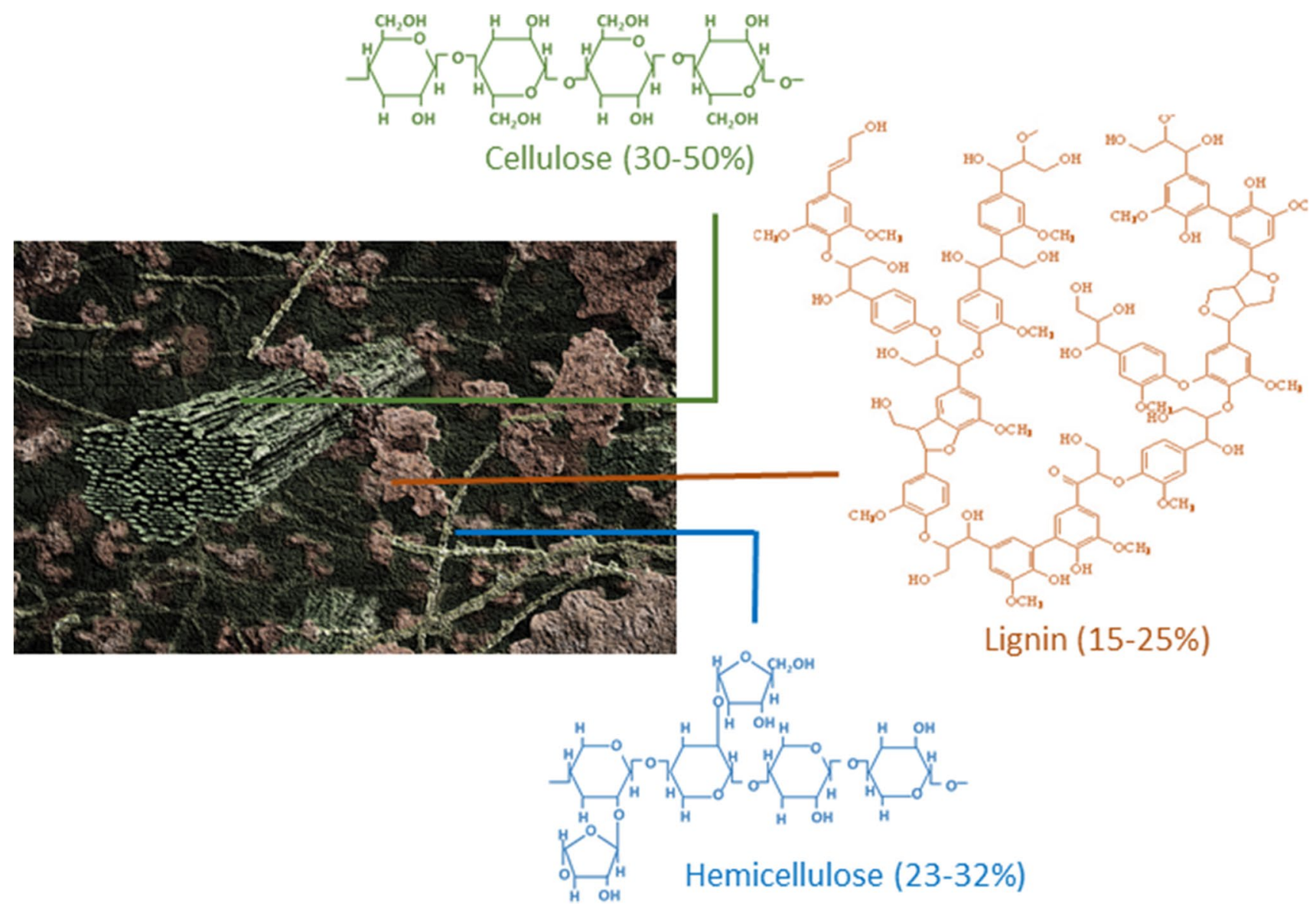

Fig. 1 Graphical representation of the principal plant cell wall components (modified from Langan et al. 2014) 
The percentages of these compounds vary in different plants and even in different parts of the same plant. In addition to these three major polymers, plant material also contains proteins, water-soluble compounds, lipids, minerals and, in many cases, reserve polysaccharides like starch.

Cellulose and hemicellulose are the two most abundant polysaccharides on Earth. Due to their natural abundance and renewable character, these polymers are target substrates for obtaining fuels and added-value products that replace those currently derived from fossil resources (Tuck et al. 2012).

Cellulose is a structural homopolysaccharide, made of long linear chains of $\beta$-(1-4)-D-glucopyranose. The regular arrangement of the hydroxyl groups along the cellulose chain leads to the formation of H-bridges, and therefore to a fibrillary and recalcitrant structure with crystalline properties. However, some regions, known as amorphous cellulose, lack crystallinity within this extremely ordered structure (Meng and Ragauskas, 2014). Hemicellulose is the generic name of several plant cell wall heteropolysaccharides that share their branched nature and the presence of $\beta-(1,4)$ linkages in their main backbone (Méndez-Líter et al. 2021). Based on their monosaccharide composition, they are denominated xylans, mannans, xyloglucans, mixedlinkage $\beta$-glucans, and their variants, which differ in their backbone and branches, and in the type and distribution of glycosidic bonds. The presence of one or another polysaccharide depends on the type of plant, but the most abundant non-cellulosic polysaccharides are xylans. They have a main backbone of $\beta$ - $(1,4)$-xylopyranose, substituted by residues of arabinose (arabinoxylans) or glucuronic acid (glucuronoxylans) and, in many instances, they are acetylated. Thus, the complexity of these polymers is high, as well as the number of enzymes required for their full degradation. The third major component of lignocellulose is lignin, a heterogeneous polymer formed by random polymerization of monolignols (phenylpropane-type units) bound by $\mathrm{C}-\mathrm{C}$ and $\beta-1,4$ linkages. These linkages are extremely resistant to degradation and therefore lignin acts as a natural barrier, responsible for conferring resistance and protection on plants.

Fungi play a pivotal role in the breakdown of organic matter and are responsible along with bacteria for recycling nitrogen, carbon, and other vital elements from plant biomass. Their metabolic versatility mostly relies in their ability to produce and secrete an immense enzymatic arsenal in the presence of substrates, which induce the expression of the genes involved in their degradation. Live or decayed wood and vegetal debris in soils are among the multiplicity of habitats colonized by fungi, and for this reason, a good number of fungal species and/or their enzymes have been successfully applied for the biotransformation of vegetal residues in different ways. They have developed their action according to two general patterns. White-rot fungi have a versatile multienzyme system that allows simultaneous degradation of cellulose and lignin or preferential degradation of the latter, while brown-rot fungi cause a preferential removal of cellulose leaving a brown-colored lignin residue, mostly linked to non-enzymatic oxidative mechanisms based on Fenton reaction to generate hydroxyl radicals, which attack plant cell walls via powerful oxidation reactions (Eastwood et al. 2011; Martínez et al. 2005). Thus, the knowledge on the mechanisms used by these fungi, which are able to degrade especific plant cell wall components, is determinant to design sustainable processes for valorization of both the sugar and aromatic units from lignocellulose.

Deconstruction of plant cell wall and removal of lignin are key steps for the biotechnological use of plant polysaccharides, and at the industrial scale this is done by chemical or physicochemical methods (Brodeur et al. 2011). Biodegradation of lignin by fungi (Salvachúa et al. 2013) and enzymatic delignification of lignocellulose (Martínez et al. 2009) are also possible approaches, but they are slow or expensive treatments. Once lignocellulose has lost its original structure, the degradation of polysaccharides is mainly accomplished by a specific pool of microbial glycosyl hydrolases: cellulases and hemicellulases. In the last decade, it has been shown that some accessory enzymes are co-regulated or co-expressed by microbes together with these essential enzymes during growth on cellulosic substrates (Sørensen et al. 2013). These proteins are oxidoreductases, such as lytic polysaccharide monooxygenases or cellobiose dehydrogenases, that work synergistically with hydrolases expanding their catalytic potential, reducing crystallinity and/or polysaccharides length by oxidative cleavage (Glass et al. 2013; Martínez, 2016).

\section{Glycoside hydrolases}

As mentioned above, the microbial enzymes responsible for the hydrolysis of plant polysaccharides in nature are included within this broad group of proteins, also called glycosidases or glycosyl hydrolases, and commonly abbreviated as GH. They are defined as a widespread group of enzymes that hydrolyses the glycosidic bond between two or more carbohydrates or between a carbohydrate and a noncarbohydrate moiety (Lombard et al. 2014). Specifically, the GHs active on polysaccharides are $O$-glycosidases, classified in the group EC 3.2.1 together with $S$-glycosidases, while $N$-glycosidases belong to EC 3.2.2. Since the enzymes acting on the same carbohydrate may display very different structures, an alternative classification to that provided by the Enzyme Commission was proposed in the 1990s leading to creation of the Carbohydrate-Active Enzymes Database (www.CAZy.org) that groups these proteins in families according to their sequence and, therefore, to their structure 
(Lombard et al. 2014). Hydrolysis occurs through a general acid catalysis mechanism and involves two amino acid residues, generally aspartic and/or glutamic acid, that act as a proton donor and a nucleophile/base, respectively (Davies and Henrissat, 1995). Depending on the spatial position of these catalytic residues, the hydrolysis can proceed in a single step, releasing a sugar product whose anomeric configuration is opposite to that of its precursor (inverting $\mathrm{GHs}$ ), or happen in two phases to give a carbohydrate that maintains the same anomeric configuration (retaining GHs). Due to their diversity, the structural features of glycosyl hydrolases are hardly generalizable, excepting the topology of their active site that can group them as having the groove, tunnel or pocket shapes (Juturu and Wu, 2014; Lynd et al. 2002).

Notably, some retaining GHs can catalyze transglycosylation reactions, transferring one sugar unit to a nucleophilic acceptor different to water under specific conditions. Thus, the transglycosylation activity of these GHs leads to the formation of a new glycosidic linkage (Nieto-Domínguez et al. 2020) and makes them attractive synthetic tools, opening the possibility of creating novel glycoconjugates or synthetic oligosaccharides (Danby and Withers, 2016). A scheme of hydrolysis and transglycosylation catalyzed by retaining GHs is presented in Fig. 2.

\section{Fungal cellulases and hemicellulases}

Cellulases and hemicellulases are generally inducible proteins (Lynd et al. 2002; Vaishnav et al. 2018) secreted to the medium in the presence of substrates of lignocellulosic origin (or their products) when the microorganism lacks simple sugars to metabolize. Despite the huge number of existing GH families in the CAZy database, those produced in fungal cellulolytic cocktails usually fit in to families $1,3,5,6,7$, 12, and 45 (Payne et al. 2015).

The main GHs involved in enzymatic degradation of cellulose and xylan are represented in Fig. 3. The enzymatic hydrolysis of cellulose occurs mainly thanks to the concerted and synergistic action of three types of cellulases (Thapa et al. 2020): endo- $\beta-1,4$-glucanases (EG or endoglucanases), $\beta$-1,4-exoglucanases ( $\mathrm{CBH}$, cellobiohydrolases, exocellobiohydrolases, or exocellullases), and $\beta$-1,4-glucosidases. As represented in Fig. 3A, endo- $\beta-1,4$-glucanases (EC 3.2.1.4) are non-processive cellulases that randomly cleave internal $\beta-1,4-O$-glycosidic bonds in amorphous regions of the polysaccharide. The large cellulose chains are split in shorter fragments creating new ends, where reducing- and nonreducing-end cellobiohydrolases (EC 3.2.1.91), which are processive enzymes, develop their catalytic action. CBHs release soluble oligosaccharides (mostly cellobiose) that are finally converted into glucose molecules by $\beta$-glucosidases (EC 3.2.1.21).

As occurred with cellulose, hemicellulose depolymerization involves the join and synergistic action of a set of enzymes (Fig. 3B). In the case of xylan, the most abundant hemicellulose in cereals and hardwoods (Polizeli et al. 2005), two enzymes are the main players: endo- $\beta$ 1,4-xylanases (EC 3.2.1.8) hydrolyzing $\beta-1,4$ linkages of the xylan backbone and $\beta$-xylosidases (EC 3.2.1.37) act in the non-reducing end of the oligosaccharides generated by endoxylanases, releasing monomeric xylose (Shallom and Shoham, 2003).

Trichoderma reesei (currently named Hypocrea jecorina) is the most used microorganism for industrial production of cellulases. This filamentous fungus and its enzymes have been the subject of numerous studies aimed at improving the production of cellulases and their catalytic properties.
Fig. 2 Scheme of hydrolysis and transglycosylation catalyzed by retaining glycosyl hydrolases

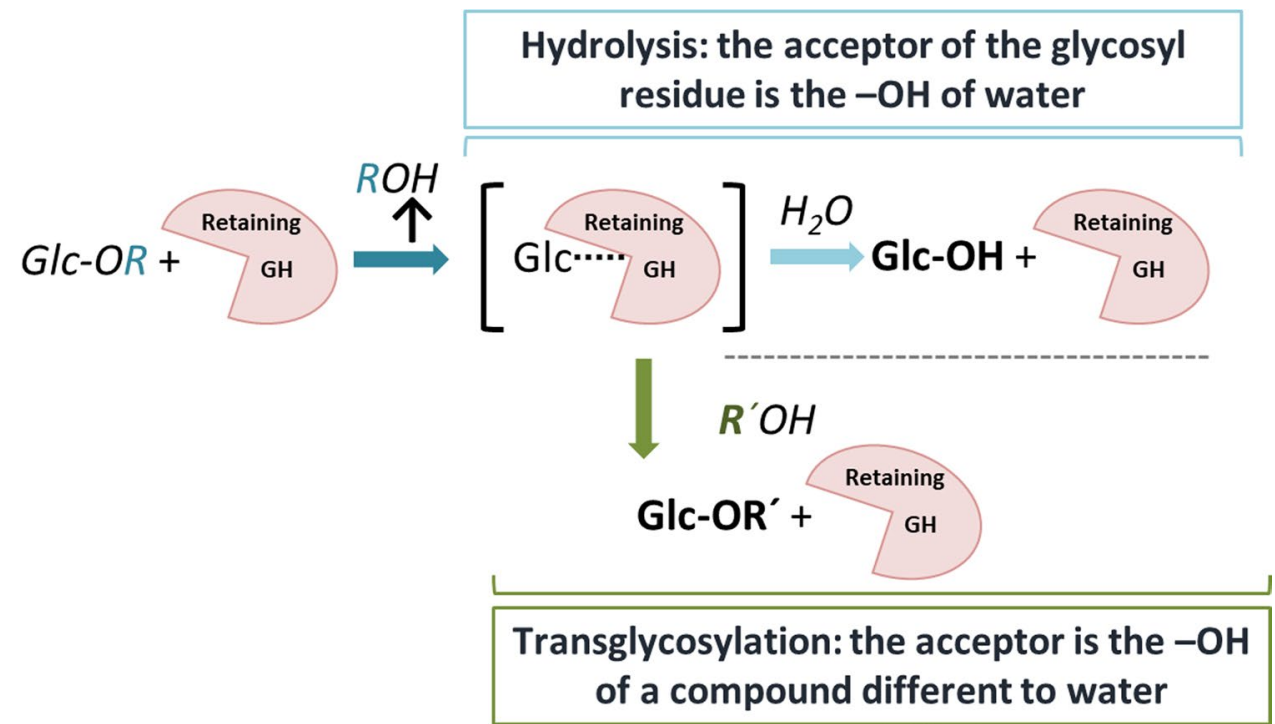


Fig. 3 Composition and degrading enzymes of $\mathbf{A}$ - cellulose; B-, xylan. Arrows point at bonds hydrolysed by the corresponding enzymes

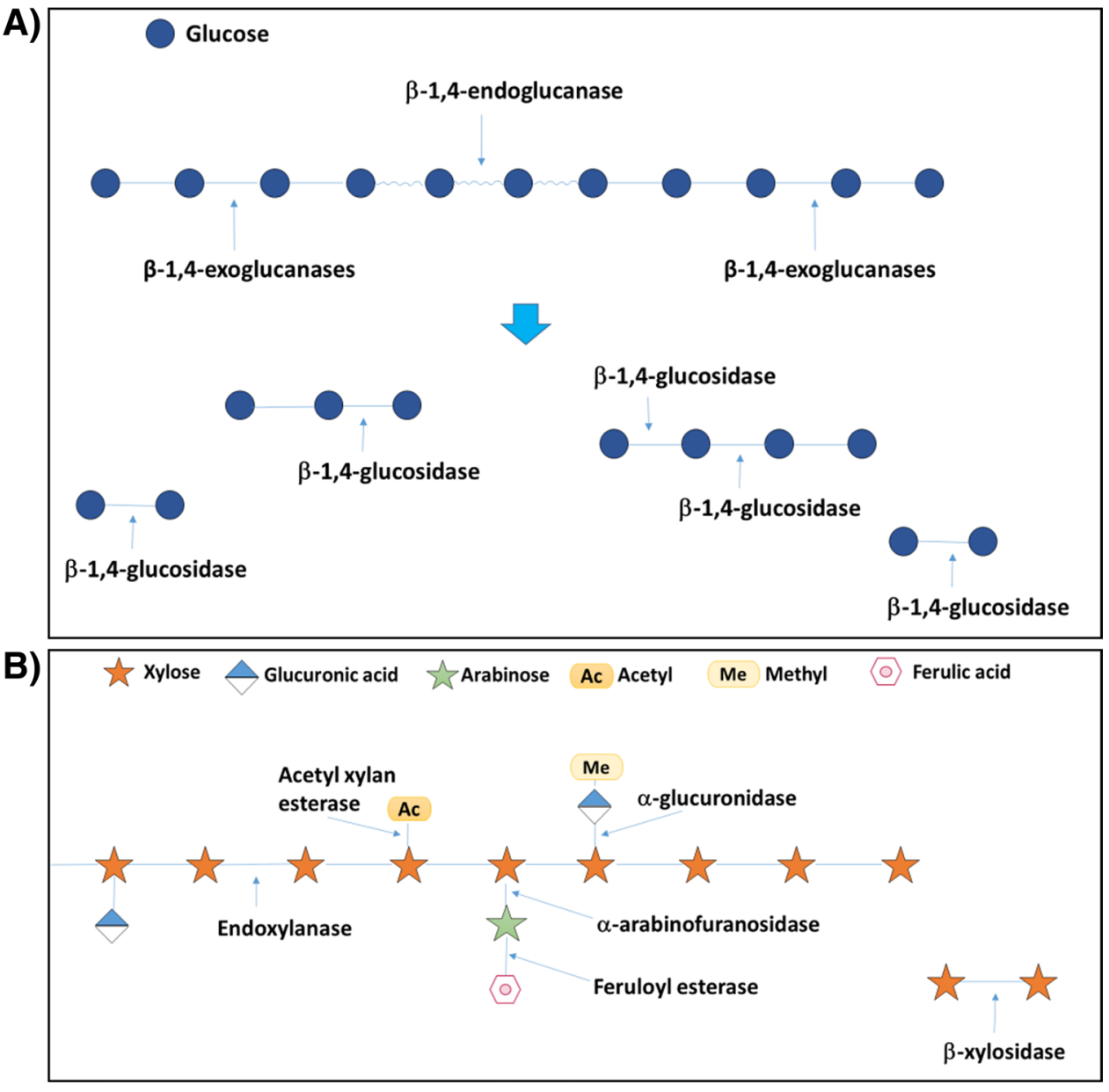

However, several reasons support that this species is not the best choice for producing cocktails for the degradation of plant biomass. On the one hand, T. reesei secretes low levels of $\beta$-glucosidases, which are essential enzymes to complete the degradation of cellulose to glucose, and in addition, they are easily inhibited by glucose. On the other hand, a study published in 2008 revealed that its genome encodes fewer cellulases and hemicellulases than any of the fungi sequenced at that time capable of hydrolyzing plant cell wall polysaccharides (Martinez et al. 2008). These issues fostered the research on alternative producers of robust enzyme cocktails to fulfill the industry demands. Although effective commercial preparations are available for cellulose hydrolysis (Table 1), there is still need and opportunity to enhance the saccharification efficiency, and the research on alternative fungal producers of robust enzyme cocktails, especially those rich in $\beta$-glucosidase activity, remain a focus of interest.

For example, some species of Penicillium and Talaromyces are more efficient than $T$. reese $i$ and give better yields of monosaccharides from biomass because they release a battery of hemicellulases along the whole cellulase pool (de Eugenio et al. 2017; Liu et al. 2013; Yang et al. 2018).
Table. 1 Some commercial preparations of fungal cellulases (Vaishnav et al. 2018)

\begin{tabular}{|c|c|c|}
\hline Preparation & Manufacturer & Fungal source \\
\hline N-50010 & Novozymes (Denmark) & A. niger \\
\hline N-188 & Novozymes & A. niger \\
\hline Celluclast $1.5 \mathrm{~L}$ & Novozymes & T. reesei \\
\hline Celtec2 & Novozymes & T. reesei \\
\hline Celtec3 & Novozymes & T. reesei \\
\hline Biocellulase A & Ques Intl. (USA) & A. niger \\
\hline Biocellulase Tri & Ques Intl & A. niger \\
\hline GC 220 & Genencor (USA) & $\begin{array}{l}\text { T. longibrachiatum/T. } \\
\text { reesei }\end{array}$ \\
\hline GC 440 & Genencor & $\begin{array}{l}\text { T. longibrachiatum/T. } \\
\text { reesei }\end{array}$ \\
\hline GC 880 & Genencor & $\begin{array}{l}\text { T. longibrachiatum/T. } \\
\text { reesei }\end{array}$ \\
\hline Accelerase 1500 & Genencor & T. reesei \\
\hline Rovabio & Adisseo (France) & P. funiculosum \\
\hline Cellulase AP30K & Amano enzyme (Japan) & A. niger \\
\hline Cellulase TAP106 & Amano enzyme & T. viride \\
\hline
\end{tabular}


Therefore, the interest in microorganisms acting on the major plant polysaccharides and contributing to the integral use of sugars in plant biomass is still a hot spot.

\section{Talaromyces amestolkiae and its enzymes as biotechnological tools for valorization of plant cell wall polysaccharides}

Talaromyces is an holomorphic genus with a very close taxonomical relatedness with Penicillium, to which many of its asexual forms belonged in the past (Samson et al. 2011). Several of its species, like Talaromyces cellulolyticus (Fujii et al. 2015), Talaromyces pinophilus (Li et al. 2017), or Talaromyces verruculosus (Goyari et al. 2015), have shown to be excellent degraders of plant polysaccharides.

For some years, our group has been working with the strain IJFM A795 of T. amestolkiae, isolated from cereal residues and selected from a fungal screening, for its ability to produce high levels of cellulases (de Eugenio et al. 2017). When this species was cultured in Mandels medium with Avicel (microcrystalline cellulose) as carbon source, the total extracellular cellulase activity was comparable to those obtained in two isolates of $T$. reesei selected in the same screening (Fig. 4). Therefore, the genome of this fungus was sequenced in order to explore in depth the real potential of its cellulolytic system.

Genome analysis revealed that the number of genes encoding for putative enzymes implicated in plant cellwall degradation was much higher than those from $T$. reesei and other cellulolytic fungi, confirming the capability of this fungus for saccharification of plant biomass (de Eugenio et al. 2017).

As the expression of most genes involved in lignocellulose degradation is inducible, the response of $T$. amestolkiae to different carbon sources was evaluated by differential proteomics. The fungus was cultivated for 7 days in the same basal medium, but containing glucose (control), Avicel, steam-exploded wheat straw slurry, or beechwood xylan. After monitoring the main lignocellulolytic activities produced in each condition, the four 7-day-old secretomes were subjected to differential shotgun proteomic analysis (de Eugenio et al. 2017). The proteomics data revealed that the complexity of the different secretomes runs in parallel with that of the polymers used as inductors, with 184 proteins identified in the xylan cultures, 144 in slurry, and 104 in Avicel. Further analysis revealed that 44 proteins were exclusively produced in xylan medium, 21 in Avicel and 15 in wheat straw slurry.

\section{A)}

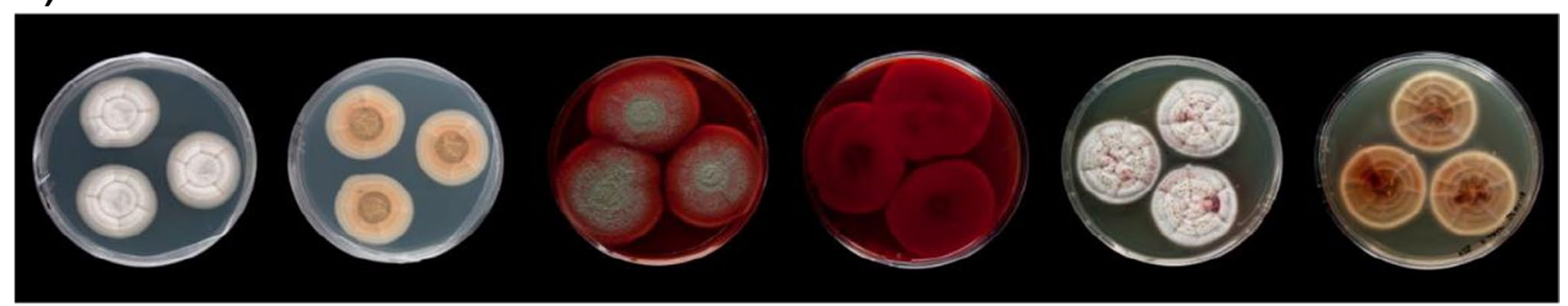

B)

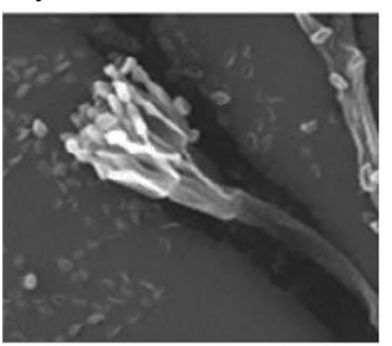

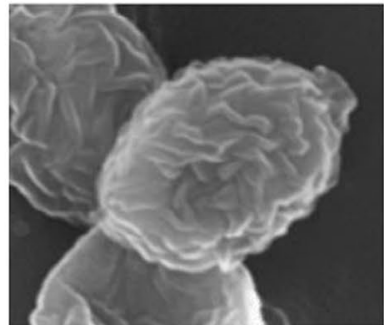

Fig. 4 Morphological identification of T. amestolkiae and comparison of the global cellulase activity (using Avicel as substrate) secreted by the three main producers found in the screening. A-From left to right, front, and back side view of the fungal colony in Czapek yeast
C)

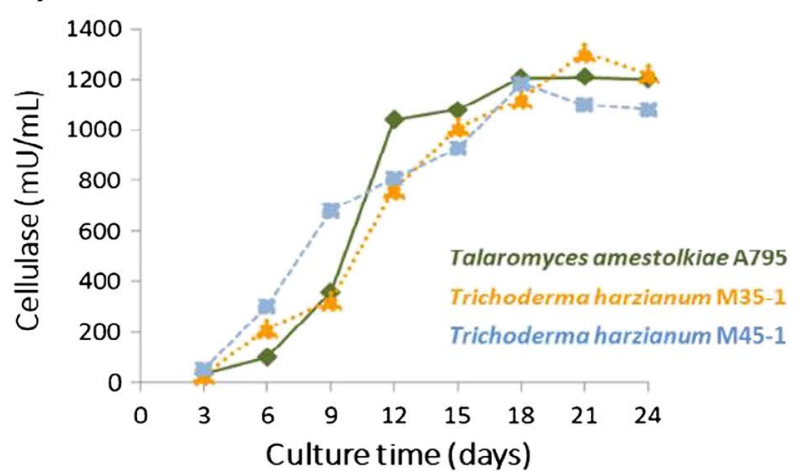

agar, Malt extract agar, and Yeast extract sucrose agar. B-SEM image of conidiophores and conidia. $\mathbf{C}$-Time course of total cellulase production (adapted from Gil-Muñoz 2015) 
Many proteins were identified from this analysis by identity search against an in-house specific database of the $T$. amestolkiae genome (10,408 sequences, 5,662,098 residues). Some of them resulted to be specific or overexpressed in a given condition, and the three polymeric inducers caused the secretion of enzyme pools active on both cellulose and hemicellulose. These data confirmed the huge potential of this isolate to produce enzyme cocktails with all the activities required for the integral treatment of plant polysaccharides.

Since then, several glycosyl hydrolases from this fungus have been purified, characterized, and produced heterologously, to study and/or modulate their activity and to investigate feasible biotechnological applications. The more interesting enzymes characterized from this strain and their applications will be detailed in the next sections.

\section{$\beta$-glucosidases from T. amestolkiae: enzymes with unique characteristics}

The initial work with T. amestolkiae focused on the isolation and characterization of B-glucosidases, because they have been repeatedly pointed out as the main bottleneck for saccharification of plant biomass (Sørensen et al. 2013). The high levels of this activity detected in all secretomes, regardless of the carbon source (de Eugenio et al. 2017), correlated perfectly with the 24 hypothetical encoding genes identified by homology search using other cellulolytic species as reference: $5 \mathrm{~B}$-glucosidases from GH1, 18 from $\mathrm{GH} 3$, and 1 from GH5. Analysis of the secretomes released in those media revealed that 6 different $B$-glucosidases were produced in Avicel and glucose media, 8 in slurry and 10 in xylan cultures. Two GH3 proteins were the major $\beta$-glucosidases detected in these analyses, which resulted promising since this family is considered to include the B-glucosidases with better catalytic efficiency, although their glucotolerance is typically low (Cao et al. 2015). Protein identification disclosed that one of them was BGL-2 (Méndez-Líter et al. 2017), which was highly represented only in the medium with Avicel $(70 \%$ of the total B-glucosidases of the secretome), suggesting that its production was strongly induced by cellulose. The other GH3 protein was the most abundant in media containing slurry, glucose, or xylan $(72,55$, and $45 \%$ of the total B-glucosidases, respectively), and showed to be BGL-3. Since the secretion of $\beta$-glucosidases in a medium with glucose as carbon source is uncommon, the time course of recombinant BGL-3 production was studied, revealing that the enzyme was released after glucose depletion, under carbon starvation (Méndez-Líter et al. 2018). In addition, one $\beta$-glucosidase from family $\mathrm{GH} 1$, detected in low amounts in all conditions assayed, was identified as BGL-1. The native BGL-1 was not completely studied due to its poor production yield, but GH1 proteins are interesting from an industrial perspective because most characterized glucose-tolerant $\beta$-glucosidases belong to this family (Singhania et al. 2013).

As the three $\beta$-glucosidases were interesting for one or another reason, the $b g l l, b g l 2$, and bgl3 genes were expressed in the yeast Pichia pastoris with the aim of improving their production levels and to complete their characterization. The main physicochemical features of the recombinant proteins are summarized in Table 2 and their kinetic properties against synthetic ( $p$-nitrophenyl- $\beta$-Dglucopyranoside, $p$ NPG) and natural substrates (cellooligosaccharides) are presented in Table 3.
Table. 2 Physicochemical properties of the recombinant $\beta$-glucosidases of $T$. amestolkiae produced in P. pastoris (data from Méndez-Líter et al. 2017, 2018, 2020)

Table. 3 Kinetic constants of the recombinant $\beta$-glucosidases of T. amestolkiae produced in $P$. pastoris (data from MéndezLíter et al. 2017, 2018, 2020)

\begin{tabular}{lllllll}
\hline Enzyme & $\begin{array}{l}M_{\mathrm{w}}(\mathrm{kDa}) \\
\text { MALDI-TOF }\end{array}$ & Quaternary structure & Glyco-protein & $\begin{array}{l}\text { Opt } \\
\mathrm{pH}\end{array}$ & $\begin{array}{l}\text { Opt } \\
\mathrm{T}\left({ }^{\circ} \mathrm{C}\right)\end{array}$ & Family \\
\hline BGL-1 & 88.1 & Monomer & Yes & 4.0 & 60 & $\mathrm{GH} 1$ \\
BGL-2 & 102.1 & Monomer & Yes & 4.0 & 60 & GH3 \\
BGL-3 & 107.0 & Dimer & Yes & 4.0 & 70 & GH3 \\
\hline
\end{tabular}

\begin{tabular}{|c|c|c|c|c|c|c|c|c|c|}
\hline \multirow[b]{2}{*}{ Substrate } & \multicolumn{3}{|l|}{ BGL1 } & \multicolumn{3}{|l|}{ BGL2 } & \multicolumn{3}{|l|}{ BGL3 } \\
\hline & $\boldsymbol{K}_{\mathrm{m}}$ & $\boldsymbol{k}_{\mathrm{cat}}$ & $\boldsymbol{k}_{\mathrm{cat}} / \boldsymbol{K}_{\mathrm{m}}$ & $\boldsymbol{K}_{\mathrm{m}}$ & $\boldsymbol{k}_{\text {cat }}$ & $\boldsymbol{k}_{\mathrm{cat}} / \boldsymbol{K}_{\mathrm{m}}$ & $\boldsymbol{K}_{\mathrm{m}}$ & $\boldsymbol{k}_{\text {cat }}$ & $\boldsymbol{k}_{\mathrm{cat}} / \boldsymbol{K}_{\mathrm{m}}$ \\
\hline & $(\mathrm{mM})$ & $\left(\mathrm{s}^{-1}\right)$ & $\left(\mathrm{mM}^{-1} \cdot \mathrm{s}^{-1}\right)$ & $(\mathrm{mM})$ & $\left(\mathrm{s}^{-1}\right)$ & $\left(\mathrm{mM}^{-1} \cdot \mathrm{s}^{-1}\right)$ & $(\mathrm{mM})$ & $\left(\mathrm{s}^{-1}\right)$ & $\left(\mathrm{mM}^{-1} \cdot \mathrm{s}^{-1}\right)$ \\
\hline$p \mathbf{N P G}$ & 3.36 & 898 & 267 & 0.19 & 444 & 2337 & 0.14 & 1359 & 9707 \\
\hline Cellobiose & 20.36 & 138 & 7 & 1.11 & 630 & 568 & 0.48 & 1594 & 3322 \\
\hline Cellotriose & 19.39 & 196 & 10 & 1.87 & 617 & 330 & 0.80 & 983 & 1228 \\
\hline Cellotetraose & 17.62 & 277 & 16 & 0.92 & 580 & 630 & 0.35 & 1330 & 3800 \\
\hline Cellopentaose & 12.41 & 260 & 21 & 0.71 & 671 & 945 & 0.32 & 1457 & 4453 \\
\hline Cellohexaose & 9.18 & 218 & 24 & 0.51 & 405 & 794 & 0.57 & 1334 & 2340 \\
\hline
\end{tabular}


The three purified enzymes from the culture supernatants of P. pastoris were glycoproteins containing around $20 \%$ of carbohydrates. In all cases, the recombinant enzymes showed higher glycosylation degree that native proteins but it does not affect significantly their physicochemical and kinetic properties. BGL-1 is a monomeric protein, with the lowest molecular mass among the three BGLs studied. The extracellular activity secreted in P. pastoris was outstanding $(75 \mathrm{U} / \mathrm{mL})$, indicating the overexpression of $b g l l$ in this system (Méndez-Líter et al. 2020). The monomeric units of BGL-2 and 3 have similar molecular mass, but BGL-3 showed to be a dimer when analyzed under non-denaturing conditions (Méndez-Líter et al. 2017, 2018). Their optimum $\mathrm{pH}$ of 4.0 and their stability between $\mathrm{pH} 4.0$ and 7.0 are similar to those described for most fungal $\beta$-glucosidases (Bhiri et al. 2008; Chávez et al. 2006; Ramani et al. 2012).

On the other hand, few studies describe high $\beta$-glucosidase activities on natural substrates as cellobiose or cellooligosaccharides, despite these are their typical saccharification substrates (Singhania et al. 2013). The assessment of the activity of the three BGLs on cellooligosacharides from 2 to 6 glucose units confirmed that they all acted on cellobiose and their efficiency varied with the chain length (Table 3). Despite the low hydrolytic efficiency of BGL-1 on the substrates assayed (Table 3 ), this enzyme showed an outstanding glucotolerance, and its inhibition constant against glucose $(K i=3.78 \mathrm{M})$ is among the highest described to date (Méndez-Líter et al. 2020). This work also revealed that BGL-1 is a versatile enzyme, since it had higher hydrolytic activity on sophorose ( $\beta-1,2$ disaccharide of glucose) than on cellobiose ( $\beta-1,4$ disaccharide of glucose). The versatility of other GH1 proteins has already been reported (Heins et al. 2014).

Regarding BGL-2, its most interesting structural feature is that it has a Cellulose Binding Domain (CBD) (MéndezLíter et al. 2017). This is a frequent trait in enzymes that act on the cellulose polymer but not in $\beta$-glucosidases, where the biological meaning of such structure is less evident at first glance. The functionality of the BGL-2 CBD was investigated, after expressing the complete protein and its truncated form (without CBD) in P. pastoris, evaluating the capacity of both isoforms to bind to different polysaccharides. The results from this test confirmed that only the entire protein binds to cellulose, which indicates that the CBD could play an important role in attaching to natural cellulosic substrates (Méndez-Líter et al. 2017). The recombinant BGL-2 was much more efficient than BGL-1 hydrolyzing cellobiose and other short oligosaccharides (Table 3 ).

Finally, the enzyme BGL-3 produced in T. amestolkiae cultures when glucose was exhausted (Méndez-Líter et al. 2018), was purified, characterized, and recombinantly obtained in $P$. pastoris. It was the most active of the $T$. amestolkiae enzymes hydrolyzing cellooligosaccharides, with catalytic efficiencies one or two magnitude orders higher than BGL-2 and BGL-1, respectively (Table 3). In fact, the efficiency of BGL-3 on cellobiose hydrolysis was only surpassed by that of the $\mathrm{rBgl} 4$ produced by Penicillium funiculosum $\left(\mathrm{kcat} / \mathrm{Km}=3610.4 \mathrm{mM}^{-1} \cdot \mathrm{s}^{-1}\right.$ ) (Ramani et al. 2015), both exceeding by tenfold those described for most of the known fungal $\beta$-glucosidases. Another characteristic of BGL-3 is its wide substrate specificity, acting also on disaccharides different to cellobiose as laminaribiose ( $\beta$-1,3-linked glucose) and gentiobiose ( $\beta$-1,6- linked glucose), and on laminarin, a $\beta$-1,3-glucan from marine algae of the genus Laminaria. This polysaccharide is specifically depolymerized by strict laminarinases, and the activity of BGL-3 enzymes on this substrate may be due to the strong similarity of the amino acids involved in substrate binding in these enzymes.

\section{Hemicellulases produced by T. amestolkiae}

The studies of the secretomes released by T. amestolkiae growing with Avicel, slurry, or xylan as carbon sources demonstrated that hemicellulases are produced as a response to the inducers, but not in the control medium with glucose (de Eugenio et al. 2017), probably because this monosaccharide is a strong repressor of the hemicellulolytic metabolism (Chávez et al. 2006). However, the maximal levels of xylanolytic enzymes were obtained in the medium with beechwood xylan, where these biocatalysts constituted about $16 \%$ of the total secreted proteins. GH10 and/or GH11 endoxylanases were among the 10 most abundant proteins detected with the three polymeric inducers, while a GH3 $\beta$-xylosidase was identified only in cultures containing xylan, which agrees with previous reports (Hori et al. 2011; Nieto-Domínguez et al. 2015).

With this preliminary information, the native GH3 $\beta$-xylosidase BxTW1 and the GH11 endoxylanase XynM were produced in media containing 1-3\% xylan (w/v), selecting $2 \%$ as the best concentration of the inducer, and both enzymes were purified before further analysis (NietoDomínguez et al. 2015; 2017a). Their main characteristics are detailed in Table 4.

The endoxylanase XynM is a monomeric protein of low molecular mass and very selective towards xylan (NietoDomínguez et al. 2017b), which are characteristics common to GH11 xylanases (Polizeli et al. 2005). The catalytic constants of the enzyme, as well as its optimal pH and temperature, are comparable to those reported in endoxylanases from Penicillium and Talaromyces (Chávez et al. 2006), and it maintained over $60 \%$ stability across $\mathrm{pH}$ values between 2.2 and 9 after $72 \mathrm{~h}$.

On the other hand, the $\beta$-xylosidase BxTW1 (NietoDomínguez et al. 2015) is a GH3 retaining enzyme with a 
Table. 4 Physicochemical properties of the main xylanases produced by $T$. amestolkiae (Nieto-Domínguez et al. 2015; 2017a)

\begin{tabular}{lcllllll}
\hline Enzyme & $\begin{array}{l}M_{\mathrm{w}}(\mathrm{kDa}) \\
\text { MALDI-TOF }\end{array}$ & Quaternary structure & Glyco-protein & $\begin{array}{l}\mathrm{pI} \\
\mathrm{pH}\end{array}$ & $\begin{array}{l}\mathrm{Opt} \\
\mathrm{T}\left({ }^{\circ} \mathrm{C}\right)\end{array}$ & Family \\
\hline Endoxylanase XynM & 19.8 & Monomer & Yes & 5.5 & 3.0 & 50 & GH11 \\
$\beta$-xylosidase BxTw1 & 102.3 & Dimer & Yes & 7.6 & 3.0 & 70 & GH3 \\
\hline
\end{tabular}

monomeric unit of molecular mass 102,275 Da that dimerizes in aqueous solution. The protein is extremely resistant to $\mathrm{pH}$, maintaining over $80 \%$ activity between $\mathrm{pH} 2.2$ and 9 , and has a T50 value of $59.9{ }^{\circ} \mathrm{C}$ after $72 \mathrm{~h}$. Its optimum $\mathrm{pH}$ value is 3.0 , which is quite unusual among fungal $\beta$-xylosidases, as it generally ranges between 4.0 and 6.0 (Knob et al. 2010). The remarkably low optimum pH of BxTW1 might be explained by the presence of three basic residues in the surroundings of the catalytic region that may decrease the $\mathrm{p} K_{\mathrm{a}}$ of the catalytic residues by stabilizing their anionic states through the formation of salt bridges (Nieto-Domínguez et al. 2020). BxTW1 acts on xylooligosaccharides from 2 to 6 xylose units and releases oligosaccharides from xylan. Its catalytic efficiency against these xylooligosaccharides, as well as against the model substrate $p$-nitrophenyl- $\beta$-D xylopyranoside $(p \mathrm{NPX})\left(1000 \mathrm{mM}^{-1} \cdot \mathrm{s}^{-1}\right)$, was among the highest reported in the literature (NietoDomínguez et al. 2015), but it was inactive against nonxylose disaccharides.

\section{Transglycosylation catalyzed by wild-type variants of $T$. amestolkiae glycosidases}

We have already mentioned that, under kinetically controlled conditions, some glycosidases can attach a sugar unit to certain acceptors with high selectivity and sensible yields (Hermida et al. 2007). This is the case of some of the retaining glycosidases described above which, in addition to their excellent hydrolytic properties, have demonstrated their ability to synthetize tailored glycoconjugates by transglycosylation, which is among the leading targets of biocatalysis. Just to remember, retaining glycosidases break glycosidic bonds in two-steps and, in transglycosylation, an acceptor different to water is involved in the second step and a new glycoside is formed (Fig. 1).

The first enzyme from $T$. amestolkiae tested in transglycosylation reactions was the native $\beta$-xylosidase BxTW1 (Nieto-Domínguez et al. 2015). A preliminary screening of potential sugar acceptors, among a pool of alkyl and sugar alcohols, monosaccharides, and disaccharides, demonstrated the broad acceptor specificity of this enzyme, but besides its promiscuity, further experiments revealed that the reactions catalyzed by this enzyme were regioselective (Nieto-Domínguez et al. 2015). This feature, which is not common among glycosidases, is highly desirable as it makes synthesis reactions reproducible because it yields a single (or major) reaction product. Once the recombinant variant of the wild-type enzyme produced in $P$. pastoris was obtained, the preliminary screening of transxylosylation acceptors was widened to 87 compounds (flavonoids, polyols, aryl glucosides, and aminoacids), confirming that most of the substances evaluated were positive hits. This experiment indicated that the versatility of the recombinant BxTW1 was much broader than those reported for other GHs and allowed identifying several remarkable bioactive compounds whose beneficial properties may be enhanced by addition of a sugar unit (Nieto-Domínguez et al. 2016).

In view of these interesting findings, we analyzed the transglycosylation activity of the recombinant $\beta$-glucosidases from the fungus using the same type of preliminary screening as for BxTW1. In this occasion, the test included 70 potential acceptors that covered a wide variety of alcohols (sugars, sterols, phenolic compounds, or amino acids), finding that around half of the compounds tested were potentially glycosylable with BGL-2 and BGL-3, which represents an unusually high number of positive hits reactions (Méndez-Líter et al. 2019). However, for BGL-1, the transglycosylation capacity was restricted to $p$-nitrophenol sugar derivatives of glucose ( $p$ NPG), galactose ( $p$ NPGal), and xylose ( $p$ NPX) (Méndez-Líter et al. 2020).

Although promising for being a highly specific and green methodology, the synthesis of oligosaccharides and glycoconjugates using glycosidases has a main drawback. From the outset of the reaction, an equilibrium is established between transglycosylation and hydrolysis. This equilibrium gradually shifts towards hydrolysis as the concentration of the initial donor falls and that of the product increases. Thus, the process is economically unfeasible at a large-scale, since this balance between hydrolysis and synthesis is usually linked to low glycosylation yields (Danby and Withers, 2016). However, the development of engineered glycosidases with activities fully or mostly deviated to synthesis has been a major advance, increasing these yields. 


\section{Transformation of T. amestolkiae glycosidases in efficient transglycosylation tools}

The substitution of one of the two catalytic residues of retaining GHs by an inert amino acid leads to two types of mutant enzymes with reduced or null hydrolytic potential and high glycosylation activity. When the mutation affects the acid/base residue, they are denominated thioglycoligases, whereas in glycosynthase mutants, the change is introduced in the nucleophile amino acid. Thioglycoligases have a strong catalytic balance towards glycosylation, but they usually need an activated glycosyl donor and a strong nucleophile as acceptor, reducing the scope of their biotechnological applicability (Danby and Withers, 2016). On the other hand, glycosynthases use glycosyl fluoride donors as surrogates of the glycosyl enzyme intermediate and cannot degrade the formed glycosides, producing high reaction yields (Danby and Withers, 2016). They have already been applied to synthetize oligosaccharides of nitrophenyl and methylumbellyferyl sugars (Perugino et al. 2004) and oligosaccharides (Hayes and Varki, 1993; Saumonneau et al. 2016).

Very recently, the first GH3 thioglycoligase has been obtained by directed mutagenesis of the recombinant $\beta$-xylosidase BxTW1 of $T$. amestolkiae (Nieto-Domínguez et al. 2019b, 2020). Among the mutations tested, the variant BxTW1-E495A was selected primarily based on its kinetic constants, evaluating its acceptor range. The promiscuity of this mutant was surprisingly high, much higher to that of the wild-type recombinant enzyme, producing O-, N-, S-, and Se- glycosides, sugar esters, and phosphoesters. The reasons behind this extraordinarily high acceptor range are suggested to be a combination of two main factors: (i) the subsite +1 gets wider and shallower after mutation, making the acceptors approach to the catalytic center easier, and (ii) the presence in GH3 enzymes of a conserved additional acid residue in the active site. The last observation may explain why, unlike other thioglycoligases, acidic acceptors enter the active site of BxTW1E495A, and served also to justify the good activity of GH3 enzymes at considerably low $\mathrm{pH}$ values. These properties of GH3 enzyme were corroborated in the thioglycoligase variant BGL-2-E446A of the recombinant BGL-2 of $T$. amestolkiae, which was also able to produce N- S- and Oglycosides (Nieto-Domínguez et al. 2019b, 2020).

The study from Nieto-Domínguez et al. (2020) with BxTW1-E495A also disclosed that the $\mathrm{p} K_{\mathrm{a}}$ of the acceptor is the main determinant of the feasibility of glycosylation. With the data from a large screening of acceptors, the authors hypothesized that this thioglycoligase can transfer a xylose unit to any functional group whose $\mathrm{p} K_{\mathrm{a}}$ value ranges between 2 and 9. If true, the potential glycosylation acceptors for this and other GH3 thioglycoligases would be virtually unlimited which has led to propose the name of "multiligases" for this new type of enzymes.

In parallel with that revealing work, glycosynthase mutants of the recombinant $\beta$-glucosidase BGL-1 of $T$. amestolkiae were also developed and tested (Méndez-Líter et al. 2020) and, in this case, the variant BGL-1-E521G was selected. The mutant showed to have a wider acceptor range than the wild-type recombinant BGL-1, catalyzing the regioselective $\beta-1,2$ transglycosylation of several $p \mathrm{NP}$-sugars and phenolic compounds of industrial interest.

\section{Biotechnological applications of the wild-type and mutant enzymes from $T$. amestolkiae}

From the information presented so far, it can be deduced that both the native and recombinant wild-type enzymes of $T$. amestolkiae and the mutants generated in the laboratory from some of them are extraordinary tools for the biotransformation of plant biomass polysaccharides. We have explored some applications for these biocatalysts, that are outlined below.

\section{Saccharification of lignocellulosic residues to produce bioethanol}

When induced by different lignocellulosic-derived materials, the culture supernatants of T. amestolkiae are powerful enzymatic cocktails for depolymerization of pretreated agricultural and/or agroindustrial wastes. Depending on the inducer, the cocktails are enriched in specific activities, but they always have high $\beta$-glucosidase levels (De Eugenio et al. 2017). Thus, the whole secretomes were tested in saccharification of pretreated wheat straw, one of the most common wastes used at the industrial level, as supplement of a commercial basal cellulase preparation (Celluclast 1.5 L FG, from Novozymes). Comparison with other commercial $\beta$-glucosidase supplements (N50010, Novozymes) indicated that the saccharification of cellulose to glucose in this feedstock was superior with the cocktail of $T$. amestolkiae induced by cellulose. This experiment was also done using the recombinant BGL-3 as additional source of this activity, confirming to have better saccharification efficiency than the cocktail N-50010 (Méndez-Líter et al. 2018). Besides, the outstanding BGL- 3 activity on the $\beta-1,3$ polysaccharide laminarin opens the possibility of its use for saccharification of alternative glucans from algae.

Similarly, the pure recombinant BGL-2 was assayed in saccharification of a different substrate, brewers' spent grain, 
being comparable to commercial $\beta$-glucosidase cocktails (Méndez-Líter et al. 2017).

\section{Production of prebiotic xylooligosaccharides}

After purification from the supernatants of T. amestolkiae cultures induced by xylan, the native endoxylanase XynM was used to generate xylooligosaccharides (XOS) from birchwood xylan (Nieto-Domínguez et al. 2017b). The prebiotic properties of the mixture produced by enzymatic hydrolysis, whose main components were xylobiose, xylotriose, and xylotetraose, were demonstrated based on their bifidogenic capacity and from the profile of organic acids, determined by adding XOS to the fermentation medium of breast-fed children's feces.

\section{Synthesis of bioactive glycoconjugates}

The wild-type recombinant BGL-2, BGL-3, and BxTW1 have been successfully used as catalysts of the transglycosylation of several acceptors. For these reactions, some compounds with already known biological activities were selected to carry out full experiments. First, the selective synthesis of non-natural glycosides from different phenolic antioxidants was carried out using the recombinant BxTw1 to evaluate changes in their bioactivities. Xylosides from hydroxytyrosol (the main antioxidant from olive oil), hydroquinone, and catechol were successfully produced, using xylobiose as donor (Nieto-Domínguez et al. 2017a). Using macrophage and SH-SY5Y neuroblastoma cultures, the neuroprotective and oxidative stress-reducing effect of the xyloside of hydroxytyrosol was compared with those of its aglycon (hydroxytyrosol) and resveratrol, which are compounds with recognized antioxidant and neuroprotective activity. The results confirmed that this new glycoside is non-toxic, is more effective than hydroxytyrosol in decreasing the levels of intracellular reactive oxygen species, and its neuroprotective effect is considerably greater. On the other hand, the antitumor 2-(6-hydroxynaphthyl) $\beta$-D-xylopyranoside was enzymatically synthetized for the first time in a reaction catalyzed by the recombinant BxTw1 (Nieto-Domínguez et al. 2016) that used xylobiose as sugar donor. This approach constitutes an important step forward in the production of this drug, because it avoids the complex experimental design and the use of toxic reagents characteristic of its chemical synthesis, but xylobiose is quite expensive for industrial purposes. Thus, in a later work, we devised an enzyme cascade to produce this compound from beechwood or birchwood xylan, two cost-effective polysaccharides easily available from biomass. The first step in the cascade consisted in the breakage of xylan to xylooligosaccharides, catalyzed by the endoxylanase XynM and these oligosaccharides acted as sugar donors in a further transglycosylation step, catalyzed by BxTw1 (Nieto-Domínguez et al. 2019a, b).

The recombinant BxTw1 was later immobilized as magnetic crosslinked enzyme aggregates (mCLEAs) by Murguiondo et al. (2021). The activity of BxTw1-mCLEAs and the soluble enzyme were compared in the transfer of a xylose unit from $p$-nitrophenyl xylopiranoside ( $p \mathrm{NPX}$ ), an activated sugar donor, to the antioxidants hydroxytyrosol and vanillyl alcohol. Both biocatalysts showed similar profiles of transglycosylation and hydrolysis. However, the magnetic biocatalyst was easily recovered with a magnet and reused in four successive reaction cycles.

Besides xylosides, the glucosides of the phenolic antioxidants hydroxytyrosol and vanillin alcohol were synthetized in reactions catalyzed by the $\beta$-glucosidase BGL-2 (Méndez-Líter et al. 2019). The antiproliferative activity of both phenols and of their glucosides was evaluated in breast cancer cell models, confirming that the glycoconjugates were more effective and/or had safer profiles than their nonglycosylated precursors.

Nevertheless, the free or immobilized mutant enzymes showed to be the best biocatalysts to produce glycoconjugates. $\beta$-1,2-glucosylated derivatives of epigallocatechin gallate (EGCG), the most abundant catechin in green tea, known for its biological activity, as well as of several $p$ NP-sugars, have been synthesized using the glycosynthase variant BGL-1-E521G, derived from of $\beta$-glucosidase BGL-1 (Méndez-Líter et al. 2020). Similarly, the free thioglycoligase BxTW1-E495A and the mCLEAs obtained after its immobilization were assayed in the synthesis of the O-xylosides of vanillin and EGCG (Murguiondo et al. 2021). The two enzyme preparations succeeded in producing the corresponding glycoconjugates, but the mCLEAs were recovered and used four times in the same reaction, without apparent decrease of their catalytic activity.

Moreover, both the soluble BxTW1-E495A and the BxTW1-E495A-mCLEAs synthetized N-and S-xylosides, although the immobilized thioglycoligase showed to have similar or better activity, depending on the reaction. The excellent performance and recyclability of the immobilized biocatalyst, summed to the simplified purification of the products from a reaction mixture free of enzyme, opens new and promising expectations to the enzymatic synthesis of industrially relevant glycoconjugates.

\section{Concluding remarks}

Plant biomass is the main renewable feedstock for biotransformations, and the microbial enzymes that catalyze its natural degradation provide an essential biotechnological arsenal to sustainably produce fuels, chemicals, and consumer goods. The enzymatic pool secreted by fungi in the presence 
of cellulose and hemicellulose, the major polysaccharides on Earth, act synergistically to degrade these polymers to simpler sugars that are themselves value-added compounds or serve as substrates or building blocks for further bioconversions. T. amestolkiae is a good model of such fungi, releasing powerful enzyme cocktails in response to lignocellulosic inducers for their breakdown. Several biocatalysts have been purified, produced in P. pastoris, and thoroughly characterized to evaluate their main physicochemical and catalytic traits. Special emphasis should be put on the $\beta$-glycosidases purified from this species, three $\beta$-glucosidases and one $\beta$-xylosidase, and on the mutants designed for some of them, whose hydrolytic activity has been abolished or extremely reduced by directed mutagenesis. All of them have shown their efficiency in biotechnological processes like saccharification, production of prebiotics, or synthesis of glycoconjugates, contributing to the development of green alternatives for sustainable exploitation of biomass.

Acknowledgements The authors thank the Gas Chromatography and Proteomics and Genomics Services of the Margarita Salas Biological Research Center (CIB-CSIC) for their help, and the collaborators who have made this work possible. In addition, we are grateful to J. Gil-Muñoz for his contribution at the initial stages of this work. The authors thank the SusPlast-CSIC Interdisciplinary Platform for their support.

Author contribution A.P and M.J.M. conceived and designed the studies. L.E, M.N., J.M, CM. L.B. studied the enzymes and acquired the data. All authors contributed to draft the manuscript and A.P. and M.J.M substantially reviewed it.

Funding This work was funded by projects GLYSUS RTI2018093683-B-I00 (MICIU/AEI/FEDER) and RETOPROSOST-2-CM, S2018/EMT-4459 (Comunidad de Madrid). Preliminary investigations were funded by MINECO or Comunidad de Madrid in collaboration with Abengoa Research and Bioenergy.

Data availability Data of the compounds are not available from the authors.

\section{Declarations}

Ethics approval Not applicable.

Consent to participate All the authors consent for their participation in the manuscript.

Informed consent Not applicable.

Competing interests The authors declare no competing interests.

\section{References}

Aditiya HB, Mahlia TMI, Chong WT, Nur H, Sebayang AH (2016) Second generation bioethanol production: a critical review. Renew Sustain Energy Rev 66:631-653

Bar-On YM, Phillips R, Milo R (2018) The biomass distribution on Earth. Proc Natl Acad Sci U S A 115:6506-6511

Bhiri F, Chaabouni SE, Limam F, Ghrir R, Marzouki N (2008) Purification and biochemical characterization of extracellular $\beta$-glucosidases from the hypercellulolytic Pol6 mutant of Penicillium occitanis. Appl Biochem Biotechnol 149:169-182

Brodeur G, Yau E, Badal K, Collier J, Ramachandran KB, Ramakrishnan S (2011) Chemical and physicochemical pretreatment of lignocellulosic biomass: A review. Enzyme Res 2011:787532

Cao, LC, Wang, ZJ, Ren, GH, Kong, W, Li, L, Xie, W, Liu, YH (2015) Engineering a novel glucose-tolerant $\beta$-glucosidase as supplementation to enhance the hydrolysis of sugarcane bagasse at high glucose concentration. Biotechnol Biofuels 8:202

Chávez R, Bull P, Eyzaguirre J (2006) The xylanolytic enzyme system from the genus Penicillium. J Biotechnol 123:413-433

Danby PM, Withers SG (2016) Advances in enzymatic glycoside synthesis. ACS Chem Biol 11:1784-1794

Davies G, Henrissat B (1995) Structures and mechanisms of glycosyl hydrolases. Structure 3:853-859

De Eugenio LI, Méndez-Líter JA, Nieto-Domínguez M, Alonso L, Gil-Muñoz J, Barriuso J, Prieto A, Martínez MJ (2017) Differential $\beta$-glucosidase expression as a function of carbon source availability in Talaromyces amestolkiae: a genomic and proteomic approach. Biotechnol Biofuels 10:161

Demirbas A (Ed) (2010) Biorefinery, in: Biorefineries. For Biomass Upgrading Facilities. Springer-Verlag, London, UK, pp 75-92

Eastwood DC, Floudas D, Binder M, Majcherczyk A, Schneider P, Aerts A, Asiegbu FO, Baker SE, Barry K, Bendiksby M, Blumentritt M, Coutinho PM, Cullen D, de Vries RP, Gathman A, Goodell B, Henrissat B, Ihrmark K, Kauserud H, Kohler A, LaButti K, Lapidus A, Lavin JL, Lee Y-H, Lindquist E, Lilly W, Lucas S, Morin E, Murat C, Oguiza JA, Park J, Pisabarro AG, Riley R, Rosling A, Salamov A, Schmidt O, Schmutz J, Skrede I, Stenlid J, Wiebenga A, Xie X, Kuees U, Hibbett DS, Hoffmeister D, Hogberg N, Martin F, Grigoriev IV, Watkinson SC (2011) The plant cell wall-decomposing machinery underlies the functional diversity of forest fungi. Science 333:762-765

European Commission, (2015) Closing the loop - an EU action plan for the Circular Economy. Communication from the Commission to the European Parliament, the Council, the European Economic and Social Committee and the Committee of the Regions. COM/2015/0614 final

European Environmental Agency, (2018). The circular economy and the bioeconomy. EEA Report No. 8/2018

Fujii T, Koike H, Sawayama S, Yano S, Inoue H (2015) Draft genome sequence of Talaromyces cellulolyticus strain Y-94, a source of lignocellulosic biomass-degrading. Enzymes Microbiol Resour Announc 3(1): e00014-515

Geissdoerfer M, Savaget P, Bocken N, Hultink E (2017) The circular economy: a new sustainability paradigm? J Clean Prod 143:757-768

Gil-Muñoz J (2015) Estudio de las $\beta$-glucosidasas del complejo celulítico de Talaromyces amestolkiae: caracterización y aplicaciones biotecnológicas. Doctoral Thesis, Complutense University, Madrid. Available from: http://eprints.ucm.es/30175/1/T36063. pdf

Glass NL, Schmoll M, Cate JHD, Coradetti S (2013) Plant cell wall deconstruction by ascomycete fungi. Annu Rev Microbiol 67:477-498 
Goyari S, Devi SH, Bengyella L, Khan M, Sharma CK, Kalita MC, Talukdar NC (2015) Unveiling the optimal parameters for cellulolytic characteristics of Talaromyces verruculosus SGMNPf3 and its secret. J Appl Microbiol 119:88-98

Hayes BK, Varki A (1993) Biosynthesis of oligosaccharides in intact golgi preparations from rat liver Analysis of N-linked glycans labeled by UDP-[6-H-3]Galactose, CMP-[9-H-3]N-acetylneuraminic acid, and [Acetyl-H-3] acetyl-coenzyme A. J Biol Chem 268:16155-16169

Heins RA, Cheng X, Nath S, Deng K, Bowen BP, Chivian DC, Datta S, Friedland GD, D'Haeseleer P, Wu D, Tran-Gyamfi M, Scullin CS, Singh S, Shi W, Hamilton MG, Bendall ML, Sczyrba A, Thompson J, Feldman T, Guenther JM, Gladden JM, Cheng JF, Adams PD, Rubin EM, Simmons BA, Sale KL, Northen TR, Deutsch S (2014) Phylogenomically guided identification of industrially relevant GH1 $\beta$-glucosidases through DNA synthesis and nanostructure-initiator mass spectrometry. ACS Chem Biol 9:2082-2091

Hermida C, Corrales G, Cañada FJ, Aragón JJ, Fernández-Mayoralas A (2007) Optimizing the enzymatic synthesis of $\beta$-Dgalactopyranosyl-D-xyloses for their use in the evaluation of lactase activity in vivo. Bioorg Med Chem 15:4836-4840

Hori C, Igarashi K, Katayama A, Samejima M (2011) Effects of xylan and starch on secretome of the basidiomycete Phanerochaete chrysosporium grown on cellulose. FEMS Microbiol Lett 321:14-23

Juturu V, Wu JC (2014) Microbial exo-xylanases: a mini review. Appl Biochem Biotechnol 174:81-92

Knob A, Terrasan C, Carmona E (2010) beta-Xylosidases from filamentous fungi: an overview. World J Microbiol Biotechnol 26:389-407

Langan P, Petridis L, O'Neill HM, Pingali SV, Foston M, Nishiyama $\mathrm{Y}$ et al (2014) Common processes drive the thermochemical pretreatment of lignocellulosic biomass. Green Chem 16:63-68

Li CX, Zhao S, Zhang T, Xian L, Liao LS, Liu JL, Feng JX (2017) Genome sequencing and analysis of Talaromyces pinophilus provide insights into biotechnological applications. Sci Rep 7:490

Liu G, Qin Y, Li Z, Qu Y (2013) Improving lignocellulolytic enzyme production with Penicillium: from strain screening to systems biology. Biofuels 4:523-534

Lombard V, GolacondaRamulu H, Drula E, Coutinho PM, Henrissat B (2014) The carbohydrate-active enzymes database (CAZy) in 2013. Nucleic Acids Res 42:490-495

Lynd LR, Weimer PJ, Zyl WHV, Isak S (2002) Microbial cellulose utilization : fundamentals and biotechnology microbial cellulose utilization. Microbiol Mol Biol Rev 66:506-577

Martínez AT (2016) How to break down crystalline cellulose. Science 352:1050-1051

Martínez ÁT, Ruiz-Dueñas FJ, Martínez MJ, del Río JC, Gutiérrez A (2009) Enzymatic delignification of plant cell wall: from nature to mill. Curr Opin Biotechnol 20:348-357

Martínez ÁT, Speranza M, Ruiz-Dueñas FJ, Ferreira P, Camarero S, Guillén F, Martínez MJ, Gutiérrez A, Del Río JC (2005) Biodegradation of lignocellulosics: Microbial, chemical, and enzymatic aspects of the fungal attack of lignin. Int Microbiol 8:195-204

Martinez D, Berka RM, Henrissat B, Saloheimo M, Arvas M, Baker SE, Chapman J, Chertkov O, Coutinho PM, Cullen D, Danchin EGJ, Grigoriev IV, Harris P, Jackson M, Kubicek CP, Han CS, Ho I, Larrondo LF, De Leon AL, Magnuson JK, Merino S, Misra M, Nelson B, Putnam N, Robbertse B, Salamov AA, Schmoll M, Terry A, Thayer N, Westerholm-Parvinen A, Schoch CL, Yao J, Barbote R, Nelson MA, Detter C, Bruce D, Kuske CR, Xie G, Richardson P, Rokhsar DS, Lucas SM, Rubin EM, Dunn-Coleman N, Ward M, Brettin TS (2008) Genome sequencing and analysis of the biomass-degrading fungus Trichoderma reesei (syn. Hypocrea jecorina). Nat Biotechnol 26(5):553-560
Méndez-Líter JA, de Eugenio LI, Nieto-Domínguez M, Prieto A, Martínez MJ (2021) Hemicellulases from Penicillium and Talaromyces for lignocellulosic biomass valorization: A review. Bioresour Technol 324:124623

Méndez-Líter JA, De Eugenio LI, Prieto A, Martínez MJ (2018) The $\beta$-glucosidase secreted by Talaromyces amestolkiae under carbon starvation: a versatile catalyst for biofuel production from plant and algal biomass. Biotechnol Biofuels 11:123

Méndez-Líter JA, Gil-Muñoz J, Nieto-Domínguez M, Barriuso J, De Eugenio LI, Martínez MJ (2017) A novel, highly efficient $\beta$-glucosidase with a cellulose-binding domain: Characterization and properties of native and recombinant proteins. Biotechnol Biofuels 10(1):256

Méndez-Líter JA, Nieto-Domínguez M, Fernández De Toro B, González-Santana A, Prieto A, Asensio JL, Cañada FJ, De Eugenio LI, Martínez MJ (2020) A glucotolerant $\beta$-glucosidase from the fungus Talaromyces amestolkiae and its conversion into a glycosynthase for phenolic compounds glycosylation. Microb Cell Fact 19:127

Méndez-Líter JA, Tundidor I, Nieto-Domínguez M, Fernández de Toro B, González Santana A, de Eugenio LI, Prieto A, Asensio JL, Cañada FJ, Sánchez C, Martínez MJ (2019) Transglycosylation products generated by Talaromyces amestolkiae GH3 $\beta$ - glucosidases : effect of hydroxytyrosol, vanillin and its glucosides on breast cancer cells. Microb Cell Fact 18:97

Meng X, Ragauskas AJ (2014) Recent advances in understanding the role of cellulose accessibility in enzymatic hydrolysis of lignocellulosic substrates. Curr Opin Biotechnol 27:150-158

Murguiondo C, Mestre A, Méndez-Líter JA, Nieto-Domínguez M, de Eugenio LI, Molina-Gutiérrez M, Martínez MJ, Prieto A (2021) Enzymatic glycosylation of bioactive acceptors catalyzed by an immobilized fungal $\beta$-xylosidase and its multi-glycoligase variant. Int J Biol Macromol 167:245-254

Nieto-Domínguez M, de Eugenio LI, Barriuso J, Prieto A, de Toro BF, Canales-Mayordomo, A, Martínez MJ (2015) Novel pH-stable glycoside hydrolase family $3 \beta$-xylosidase from Talaromyces amestolkiae: an enzyme displaying regioselective transxylosylation. Appl Environ Microbiol 81:6380-6392

Nieto-Domínguez M, de Eugenio LI, Peñalver P, Belmonte-Reche E, Morales JC, Poveda A, Jiménez-Barbero J, Prieto A, Plou FJ, Martínez MJ (2017a) Enzymatic synthesis of a novel neuroprotective hydroxytyrosyl glycoside. J Agric Food Chem 65:10526-10533

Nieto-Domínguez M, de Eugenio LI, York-Durán MJ, Rodríguez-Colinas B, Plou FJ, Chenoll E, Pardo E, Codoñer F, Jesús Martínez M (2017b) Prebiotic effect of xylooligosaccharides produced from birchwood xylan by a novel fungal GH11 xylanase. Food Chem 232:105-113

Nieto-Domínguez M, Fernández de Toro B, de Eugenio LI, Santana AG, Bejarano-Muñoz L, Armstrong Z, Antonio MéndezLíter J, Luis Asensio J, Prieto A, Withers SG, Javier Cañada F, Jesús Martínez M (2020) Thioglycoligase derived from fungal GH3 $\beta$-xylosidase is a multi-glycoligase with broad acceptor tolerance. Nat Commun 11(1):4864. https://doi.org/10.1038/ s41467-020-18667-3

Nieto-Domínguez M, Martínez-Fernández JA, Fernández De Toro B, Méndez-Líter JA, Cañada FJ, Prieto A, De Eugenio LI, Martínez MJ (2019a) Exploiting xylan as sugar donor for the synthesis of an antiproliferative xyloside using an enzyme cascade. Microb Cell Fact 18:174

Nieto-Domínguez M, Martínez MJ, Cañada FJ, Gonzalez-Santana A, Prieto A, Asensio JL (2019b) Procedimiento para la obtención de glicoconjugados P201930082; PCT/ES2020/070079

Nieto-Domínguez M, Prieto A, Fernández de Toro B, Cañada FJ, Barriuso J, Armstrong Z, Withers SG, de Eugenio LI, Martínez MJ (2016) Enzymatic fine-tuning for 2-(6-hydroxynaphthyl) $\beta$-D-xylopyranoside synthesis catalyzed by the recombinant 
$\beta$-xylosidase BxTW1 from Talaromyces amestolkiae. Microb Cell Fact 15:171

Payne CM, Knott BC, Mayes HB, Hansson H, Himmel ME, Sandgren M, Ståhlberg J, Beckham GT (2015) Fungal cellulases. Chem Rev 115:1308-1448

Perugino G, Trincone A, Rossi M, Moracci M (2004) Oligosaccharide synthesis by glycosynthases. Trends Biotechnol 22:31-37

Polizeli MLTM, Rizzatti ACS, Monti R, Terenzi HF, Jorge JA, Amorim DS (2005) Xylanases from fungi: properties and industrial applications. Appl Microbiol Biotechnol 67:577-591

Ramani G, Meera B, Vanitha C, Rajendhran J, Gunasekaran P (2015) Molecular cloning and expression of thermostable glucose-tolerant $\beta$-glucosidase of Penicillium funiculosum NCL1 in Pichia pastoris and its characterization. J Ind Microbiol Biotechnol 42:553-565

Ramani G, Meera B, Vanitha C, Rao M, Gunasekaran P (2012) Production, purification, and characterization of a beta-glucosidase of Penicillium funiculosum NCL1. Appl Biochem Biotechnol 167:959-972

Robak K, Balcerek M (2018) Review of second generation bioethanol production from residual biomass. Food Technol Biotechnol 56:174-187

Salvachúa D, Prieto A, Vaquero ME, Martínez ÁT, Martínez MJ (2013) Sugar recoveries from wheat straw following treatments with the fungus Irpex lacteus. Bioresour Technol 131:218-225

Samson RA, Yilmaz N, Houbraken J, Spierenburg H, Seifert KA, Peterson SW, Varga J, Frisvad JC (2011) Phylogeny and nomenclature of the genus Talaromyces and taxa accommodated in Penicillium subgenus Biverticillium. Stud Mycol 70:159-183

Saumonneau A, Champion E, Peltier-Pain P, Molnar-Gabor D, Hendrickx J, Tran V, Hederos M, Dekany G, Tellier C (2016) Design of an $\alpha$-L-transfucosidase for the synthesis of fucosylated HMOs. Glycobiology 26:261-269
Shallom D, Shoham Y (2003) Microbial hemicellulases. Curr Opin Microbiol 6:219-228

Singhania RR, Patel AK, Sukumaran RK, Larroche C, Pandey A (2013) Role and significance of beta-glucosidases in the hydrolysis of cellulose for bioethanol production. Bioresour Technol 127:500-507

Sørensen A, Lübeck M, Lübeck PS, Ahring BK (2013) Fungal betaglucosidases: a bottleneck in industrial use of lignocellulosic materials. Biomolecules 3:612-631

Thapa S, Mishra J, Arora N, Mishra P, Li H, O'Hair J, Bhatti S, Zhou S (2020) Microbial cellulolytic enzymes: diversity and biotechnology with reference to lignocellulosic biomass degradation. Rev Environ Sci Biotechnol 19:621-648

Tuck CO, Pérez E, Horváth IT, Sheldon RA, Poliakoff M (2012) Valorization of biomass: deriving more value from waste. Science 337:695-699

Ubando AT, Felix CB, Chen WH (2020) Biorefineries in circular bioeconomy: A comprehensive review. Bioresour Technol 299:122585

Vaishnav N, Singh A, Adsul M, Dixit P, Sandhu SK, Mathur A, Puri SK, Singhania RR (2018) Penicillium: the next emerging champion for cellulase production. Bioresour Technol Reports 2:131-140

Yang Y, Yang J, Liu J, Wang R, Liu L, Wang F, Yuan H (2018) The composition of accessory enzymes of Penicillium chrysogenum P33 revealed by secretome and synergistic effects with commercial cellulase on lignocellulose hydrolysis. Bioresour Technol 257:54-61

Publisher's note Springer Nature remains neutral with regard to jurisdictional claims in published maps and institutional affiliations. 